\title{
Early outcome findings of treatment for transperitoneal laparoscopy-assisted pyeloplasty
}

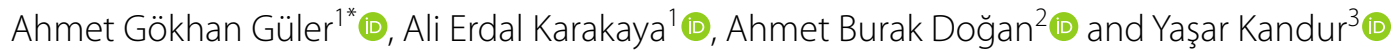

\begin{abstract}
Background: We retrospectively analyzed the initial results of laparoscopic pyeloplasty, among pediatric patients undergoing the procedure by transperitoneal access.

Methods: We retrospectively reviewed the medical records of patients who were operated with transperitoneal laparoscopy-assisted pyeloplasty at our institution between 2015 and 2020.

Results: The mean age of the 51 patients (M/F =36/15) was $59 \pm 49.8$ months. The mean preoperative renal pelvis anterior-posterior diameter was $32.9 \pm 13.4 \mathrm{~mm}$, and the relative renal function was $42 \pm 12.1 \%$ on the operation side. Forty-three (84.3\%) patients had no excretion on MAG3 examination preop. The number of patients with no excretion decreased to 10 (19.7\%) after surgery. One of them underwent a successful endopyelotomy; eight of them underwent a successful laparoscopic re-pyeloplasty. There was nothing to do in only one patient whose DRF decreased to $11 \%$. The mean anterior posterior diameter decreased significantly to $16.7 \pm 11.2 \mathrm{~mm}$ after surgery $(p<0.001)$.
\end{abstract}

Conclusion: Preoperative increased renal pelvis anterior-posterior diameter detected in our study was found to be a risk factor in the failure of transperitoneal laparoscopy-assisted pyeloplasty procedure.

Keywords: Ureteropelvic junction obstruction, Transperitoneal laparoscopic pyeloplasty, Results, MAG3

\section{Background}

Ureteropelvic junction obstruction (UPJO) is a common ureter anomaly in children. The incidence of UPJO is reportedly about 5 per 100,000 infants annually. Ureteropelvic junction obstruction is caused by intrinsic factors (IUPJO); extrinsic UPJO is rare and often occurs due to crossing aberrant vessels (CVs) [1]. It can lead to progressive hydronephrosis and renal failure [2]. The optimal time of surgery is based on a number of factors: the hydronephrosis grade, the drainage pattern of diuretic renography, the differential renal function (DRF) deterioration, and obstruction-related symptoms [3, 4]. Pyeloplasty is the main treatment method for patients with
UPJO [5]. An ideal treatment should have the highest success rate and be minimally invasive. Open pyeloplasty meets the first criterion but not the second (minimal invasiveness), whereas endourology techniques only guarantee the second one [6]. Minimally invasive techniques, both endoscopic and percutaneous ones, involving the incision of UPJ are performed with low morbidity, although they are associated with lower success rates than conventional surgery in previous studies [7]. We retrospectively analyzed the initial results of laparoscopic pyeloplasty, from the time of the first visit to the time of postoperative follow-up, among pediatric patients undergoing the procedure by transperitoneal access.

\footnotetext{
*Correspondence: drgokhanguler@hotmail.com

${ }^{1}$ Department of Pediatric Surgery, Faculty of Medicine, Sutcu Imam

University, Onikisubat, Kahramanmaras 46100, Turkey

Full list of author information is available at the end of the article
} 


\section{Methods}

We retrospectively reviewed the medical records of patients who were operated with transperitoneal laparoscopic pyeloplasty by the same surgeon for UPJO at our institution between 2015 and 2020. The diagnosis of UPJO was based on clinical symptoms and imaging studies such as renal ultrasonography (US) and Tc-99 m mercaptoacetyltriglycine (MAG3) renal scans. Surgical treatment was indicated when the patient had symptoms such as abdominal or flank pain, progressive hydronephrosis, or renal functional deterioration.

Dynamic renography (99mTc-MAG3) was obtained after an intravenous injection of $5 \mathrm{mCi}$ Tc-99 m MAG3. Images were acquired with a dual-headed E-Cam gamma camera (Siemens, Erlangen, Germany) equipped with a low-energy all-purpose parallel-hole collimator. The DRFs of the hydronephrotic kidney and the contralateral normal kidney were calculated using the geometric mean method: the square root of each kidney's backgroundsubtracted region of interest (ROI) was counted in the anterior and posterior views. The renogram patterns of patients were evaluated retrospectively and separated into two subgroups according to the excretion phase findings tissue tracer transit (TTT), namely the drainage of the renal parenchyma: kidneys with drainage were classified as pattern 1, and those with no response (obstructive pattern) were classified as pattern 2 based on visual assessment. The interpretations of the MAG3 and US were made by an experienced nuclear medicine consultant and an experienced radiologist who were blinded to all other clinical and imaging data.

At the time of data entry, demographic findings, side of UPJO (unilateral or bilateral), number of operations, cause of UPJO (intrinsic or extrinsic), preoperative and postoperative anterior-posterior diameter (APD) of renal pelvis, kidney vertical size on ultrasound examination, preoperative and postoperative MAG3 excretion patterns, and laboratory tests including routine biochemical parameters were recorded. The exclusion criteria included having neurological lesions, anatomical abnormalities of the lower urinary tract, bilateral hydronephrosis, bilateral small kidneys, horseshoe kidneys, multicystic dysplastic kidney, vesicoureteral reflux (VUR), and chronic renal failure.

For the transperitoneal approach, the exact place of these incisions was based on the location of the UPJ. The ureteropelvic junction was pulled out of the abdominal cavity, followed by performing dismembered pyeloplasty simply over a JJ stent extracorporeally. A $5 / 0$ or 6/0 polydioxanone (PDS, Ethicon, Inc., Somerville, N. J.) interrupted suture was used to perform an anastomosis between ureter and pelvis. Pelvic reduction was performed in all patients since we performed dismembered reduction pyeloplasty. The follow-up included an abdominal ultrasound, as well as a MAG-3 diuretic renal scan 3-6 months after the procedures and whenever indicated (equivocal cases) thereafter.

A successful operation was defined as a response to diuretic administration in $\mathrm{T} 1 / 2<20 \mathrm{~min}$ and/or a decrease in time of excretion in MAG-3 renography, and decrease in APD in ultrasonographical examination [8].

\section{Statistical analysis}

Study data were analyzed by SPSS (Statistical Package for Social Science) 16.0 software package. Statistical analyses were performed with Chi-squared test and MannWhitney $U$ test. A Cox regression analysis was used to assess the association between gender, age, APD, and outcome. Associations are presented as Odds ratios with their corresponding 95\% confidence intervals (CIs). The level of significance was set at $p<0.05$. The research was approved by the local ethical committee with the ethical board approval number: 2019-04-05.

\section{Results}

The mean age of the 51 patients $(M / F=36 / 15)$ was $59 \pm 6.9$ months (range 2-192 months). Thirty-four patients $(66.7 \%)$ were operated from the left side. The UPJO was caused by intrinsic stenosis in 43 $(84.3 \%)$ patients. The mean preoperative APD was $32.9 \pm 13.4 \mathrm{~mm}$, and the relative renal function was $42 \pm 12.10 .0 \%$ on the operation side. Five patients had been operated before. Forty-three (84.3\%) patients had no excretion on MAG3 examination. The mean APD decreased significantly to $16.7 \pm 11.2 \mathrm{~mm}$ after surgery $(p<0.001)$. The number of patients with no excretion decreased insignificantly to $10(19.6 \%)(p<0.001)$. The mean TTT in patients with available excretion decreased from $29.9 \pm 8.5 \mathrm{~min}$ to $15.7 \pm 4.9 \mathrm{~min}(p<0.001)$. There was no significant difference of the ipsilateral split kidney function between the pre- versus postoperative values $(44.6 \pm 10.0$ vs $44.4 \pm 11.2, p=0.854)$.

There were 17 (41\%) patients whose kidney size decreased after successful operation. Eight (15.7\%) patients had a history of urinary tract infection. The patients' demographic properties, preoperative clinical features, peri- and postoperative outcomes are presented in Table 1. The number of patients with no excretion decreased to $10(19.7 \%)$ after surgery. One of them underwent a successful endopyelotomy; eight of them underwent a successful laparoscopic re-pyeloplasty. There was nothing to do in only one patient whose DRF decreased from 11 to $6 \%$ so that the kidneys function was not preserved.

Small anastomotic leak was observed in 4 patients in the postoperative period. In these patients, the drainage 
Table 1 Preoperative and postoperative demographic properties and radiological findings

\begin{tabular}{|c|c|c|c|}
\hline & Before surgery & After surgery & $P$ \\
\hline Age at surgery, med (range), (months) & $59 \pm 6.9$ & & \\
\hline Gender (male vs female) $n(\%)$ & $36 / 15(70.5 \% / 29.5 \%)$ & & \\
\hline Laterality (right vs left) $n(\%)$ & $17 / 34(33 \% / 67 \%)$ & & \\
\hline Etiology of UPJO (intrinsic vs extrinsic) $n(\%)$ & $43 / 7(84.3 \% / 15.7 \%)$ & & \\
\hline APD before surgery, mean (mm) & $32.9 \pm 13.4$ & $16.7 \pm 11.2$ & $<0.001$ \\
\hline Split renal function, med (range), in successful operations (\%) & $44.6 \pm 10.0$ & $44.4 \pm 11.2$ & 0.854 \\
\hline Number of patients with no excretion $n(\%)$ & $43(84.3)$ & $10(19.7)$ & $<0.001$ \\
\hline Mean tissue tracer transit time in patients with available excretion (minute) & $29.9 \pm 8.5$ & $15.7 \pm 4.9$ & $<0.001$ \\
\hline
\end{tabular}

was maintained until the anastomotic leak resolved. Common postoperative complications of the surgical repair of UPJO such as urinary tract infection, urinoma, and gross hematuria were not observed in any of the patients during the six-month follow-up.

Postoperative outcomes are compared in Table 2. It was successful in 41 (80.4\%) patients and failed in 10 (19.6\%) patients. The mean age at the operation was insignificantly greater in the unsuccessful group (70.8 \pm 62.7 vs $56.1 \pm 46.7$ years, $p=0.501)$. There was no difference with respect to laterality, gender, and etiology between the successful and unsuccessful groups (Table 2). The mean APD of the successful group was significantly smaller than the unsuccessful group at both preoperative and postoperative examinations $(p=0.023, p=0.001$, respectively).

A regression analysis showed that a larger preoperative APD was a risk factor for low surgical success $(\mathrm{OR}=0.295,95 \% \mathrm{CI}-0.002-0.775, p=0.045)$. On the other hand, neither gender, age, nor laterality conferred risk of an unsuccessful operation.

\section{Discussion}

Minimally invasive procedures have emerged to reduce the morbidity observed in open surgery. As a result of a long operative period and the need for advanced laparoscopic skills, a previous meta-analysis showed that minimally invasive pyeloplasty and open pyeloplasty lead to equal success rates [9]. The first pediatric laparoscopic pyeloplasty was performed in 1995, but it has not gained much popularity worldwide, especially in the pediatric age group. However, the advent of fine needlescopic instruments has rendered intracorporeal suturing increasingly easier [10], making more pediatric urologists likely to enter the realm of minimally invasive renal reconstructive surgery. The current study shared our experience with laparoscopic-assisted pyeloplasty technique in pediatric age.

Our findings indicating a male and left side dominancy of UPJO are consistent with the previous studies $[11,12]$. Furthermore, the results of the current study demonstrated that pelvic anteroposterior diameter significantly decreased after the operation. Song et al. [12] also showed a similar decrease in patients operated with extracorporeal transperitoneal laparoscopic pyeloplasty (from $3.6 \mathrm{~cm}$ to $1.3 \mathrm{~cm}$ ).

Although several studies [12, 13] have shown an improvement in split function, we did not find any improvement. Ritchie et al. [14] showed that MAG-3 scan can provide DRF at a similar accuracy with Tc-99 m dimercaptosuccinic acid (DMSA). Thus, in order to avoid unnecessary radiation exposure and to save time, we did not use DMSA for split function. Actually, the kidney size was successfully reduced postoperatively in $41.4 \%$ of our

Table 2 Difference between successful and unsuccessful groups of patients

\begin{tabular}{llll}
\hline & $\begin{array}{l}\text { Successful surgery } \\
\mathbf{N = 4 1 ( 8 0 . 4 \% )}\end{array}$ & $\begin{array}{l}\text { Unsuccessful surgery } \\
\boldsymbol{N = 1 0 ( 1 9 . 6 \% )}\end{array}$ & $\boldsymbol{P}$ \\
\hline Age at surgery, med (range), (months) & $56.1 \pm 46.7$ & $70.8 \pm 62.7$ & $7(70)$ \\
Gender (male) & $29(70.7)$ & $660)$ & 0.501 \\
Laterality (left) & $28(68.2)$ & $8(80)$ & 0.964 \\
Etiology of UPJO (intrinsic) & $35(85.3)$ & & 0.678 \\
APPD at surgery, mean (mm) & & $41.5 \pm 22.7$ \\
Pre & $30.8 \pm 9.2$ & $26.9 \pm 16.1$ & 0.023 \\
Post & $14.2 \pm 8.1$ & 0.001 \\
\hline
\end{tabular}


patients. This factor may cause a virtual mean decrease in split function in MAG-3, even in successful operation.

Open pyeloplasty has been the gold standard for the treatment of UPJ stenosis since its establishment, having long-term success rates over 90\% [15]. The success rates of laparoscopic pyeloplasty have been comparable with those of open surgery with long-term success rates as high as $98 \%$ in recent studies $[16,17]$. Our study showed a success rate of $80.4 \%$ in terms of functional improvement in the operated unit. The degree of ureteropelvic obstruction, APD, DFR make a difference in patients who underwent open or laparoscopic surgery. Therefore, success rates may vary across studies.

Our study showed a success rate of $80.4 \%$ in terms of functional improvement in the operated unit. However, a laparoscopic re-pyeloplasty operation increased this rate to $96 \%$. These intermediate term results are thus comparable to that of open surgery, which is widely accepted as the gold standard for treatment of UPJ obstruction [2].

A larger preoperative APD was a risk factor for unsuccessful operation. Therefore, we advise open pyeloplasty for patients with large APDs. Kandur et al. showed that a large APD threshold of $20 \mathrm{~mm}$ indicates a threshold for a severe obstruction and a low DRF [18]. We similarly showed that an APD of $40 \mathrm{~mm}$ may be a risk factor for failed procedure in laparoscopic approach.

In terms of its limitations, the present study involved a retrospective cohort. The sample size of our study was also small, and thus, a follow-up study with a larger cohort is warranted.

Laparoscopic pyeloplasty has comparable functional results with those of the conventional open technique; it also proved better than the other endoluminal procedures. It is a safe and effective alternative for the treatment of pyeloureteral junction stenosis so that it can be considered by surgeons with experience in laparoscopy as the first option. However, a large APD is a risk factor for operative failure.

\section{Conclusions}

Pyeloplasty was successfully in 41 patients, but failed in 10. Renal pelvis APD was significantly higher in failed group and was a risk factor for failed attempts. Failed cases were treated with endopyelotomy or re-pyeloplasty except for one whose differential function was poorly decreased. Thus, the overall success rate with reoperations was $96 \%$. Similar to the literature data, all the failures occurred due to scar formation in the anastomosis.

\footnotetext{
Abbreviations

UPJO: Ureteropelvic junction obstruction; CVs: Crossing aberrant vessels; DRF: Differential renal function; US: Ultrasonography; TTT: Tissue tracer transit; MAG-3: Tc-99 m mercaptoacetyltriglycine; ROI: Region of interest; APD:
}

Anterior-posterior diameter; VUR: Vesicoureteral reflux; PDS: Polydioxanone; DMSA: Tc-99 m dimercaptosuccinic acid.

\section{Acknowledgements \\ None.}

\section{Authors' contributions}

All authors contributed to the preparation of this manuscript. AGG,YK, and AEK contributed to the conceptualization, design, data collection manuscript writing, and manuscript review; AEK and ABD contributed to the data analysis. All authors read and approved the final manuscript.

\section{Funding}

No funding.

\section{Availability of data and materials}

The data sets used and analyzed during the current study are available from the corresponding author on reasonable request.

\section{Declarations}

\section{Ethics approval and consent to participate}

This study was approved by the ethics committee of Kahramanmaras Sutcu Imam University with approval Number 2019/04-05. The patients provided written consent.

\section{Consent for publication}

Not applicable.

\section{Competing interests}

The authors declare that they have no competing interests.

\section{Author details}

'Department of Pediatric Surgery, Faculty of Medicine, Sutcu Imam University, Onikisubat, Kahramanmaras 46100, Turkey. ${ }^{2}$ Department of Pediatric Surgery, Faculty of Medicine, Erciyes University, Kayseri, Turkey. ${ }^{3}$ Department of Pediatrics, Faculty of Medicine, Kırıkkale University, Kirikkale, Turkey.

Received: 9 March 2021 Accepted: 11 July 2021

Published online: 22 July 2021

\section{References}

1. Ellerkamp V, Kurth RR, Schmid E, Warmann SW, Fuchs J (2016) Differences between intrinsic and extrinsic ureteropelvic junction obstruction related to crossing vessels: histology and functional analyses. World J Urol 34:577-583

2. Inagaki T, Rha KH, Ong AM et al (2005) Laparoscopic pyeloplasty: current status. BJU Int 95:102-105

3. Longpre M, Nguan A, MacNeily EA et al (2012) Prediction of the outcome of antenatally diagnosed hydronephrosis: a multivariable analysis. J Pediatr Urol 8:135-139

4. Piepsz A, Gordon I, Brock J et al (2009) Round table on management of renal pelvic dilatation in children. J Pediatr Urol 5:437-444

5. Guliev BG (2019) Laparoscopic pyeloplasty in recurrent ureteropelvic junction obstruction. Urologiia 4:16-19

6. El-Shazly MA, Moon DA, Eden CG (2007) Laparoscopic pyeloplasty: status and review of literature. J Endourol 21:673-678

7. Nadler RB, Rao GS, Pearle MS et al (1996) Acucise endopyelotomy: assessment of long-term durability. J Urol 156:1094-1097

8. Lopez-Pujals A, Leveillee RJ, Wong C (2004) Application of strict radiologic criteria to define success in laparoscopic pyeloplasty. J Endourol 18:756-760

9. Autorino R, Eden C, El-Ghoneimi A et al (2014) Robot-assisted and laparoscopic repair of ureteropelvic junction obstruction: a systematic review and meta-analysis. Eur Urol 65:430-452

10. Lee SR, Retik AB, Borer JG et al (2006) Pediatric robot assisted laparoscopic dismembered pyeloplasty: comparison with a cohort of open surgery. J Urol 175:683-687 
11. Lasmar MTC, Hilario A et al (2010) Transperitoneal laparoscopic pyeloplasty: Brazilian ınitial experience with 55 cases. Int Braz J Urol 36:678-684

12. Song SH, Lee C, Jung J et al (2017) A comparative study of pediatric open pyeloplasty, laparoscopy-assisted extracorporeal pyeloplasty, and robotassisted laparoscopic pyeloplasty. PLoS ONE 20:e0175026

13. Sharifabad PS, Hiradfar M, Shojaeian R et al (2018) Laparoscopic-assisted extracorporeal pyeloplasty: a minimally invasive approach to pediatric ureteropelvic junction obstruction. Nephro-Urol Mon 10(e83823):1-5

14. Ritchie G, Wilkinson AG, Prescott RJ (2008) Comparison of differential renal function using technetium-99m mercaptoacetyltriglycine (MAG3) and technetium-99m dimercaptosuccinic acid (DMSA) renography in a paediatric population. Pediatr Radiol 38:857-862

15. Luke $P$ (2008) The gold standard for the treatment of uncomplicated adult ureteropelvic junction obstruction. Can Urol Assoc J 2:393
16. Sundaram CP, Grubb RL 3rd, Rehman J, Yan Y, Chen C, Landman J et al (2003) Laparoscopic pyeloplasty for secondary ureteropelvic junction obstruction. J Urol 169:2037-2040

17. Moon DA, El-Shazly MA, Chang CM et al (2006) Laparoscopic pyeloplasty: evolution of a new gold standard. Urology 67:932-936

18. Kandur Y, Salan A, Guler AG et al (2018) Diuretic renography in hydronephrosis: a retrospective single-center study. Int Urol Nephrol 50:1199-1204

\section{Publisher's Note}

Springer Nature remains neutral with regard to jurisdictional claims in published maps and institutional affiliations.

\section{Submit your manuscript to a SpringerOpen ${ }^{\odot}$ journal and benefit from:}

- Convenient online submission

- Rigorous peer review

- Open access: articles freely available online

- High visibility within the field

- Retaining the copyright to your article

Submit your next manuscript at $\boldsymbol{\text { springeropen.com }}$ 